\title{
Uso de diferentes tipos de substratos e frequência variada de irrigação no crescimento inicial de pimenta biquinho (Capsicum chinense)
}

\section{Use of different substrate types and varied frequency of irrigation in the early growth of pepper pout (Capsicum chinense)}

\author{
Willians Luiz Bueno de Souza; Maira Mattar \\ Centro Universitário da Fundação Educacional de Barretos - Rua Professor Roberto Frade Monte, 389 , \\ Bairro Aeroporto - 14783-226 - Barretos (SP), Brasil -
}

\begin{abstract}
Resumo
A cultura da pimenta biquinho (Capsicum chinense) é de grande importância, tanto pelas características de rentabilidade econômica, quanto pela sua relevância social. Sendo assim, o presente estudo teve como objetivo avaliar o uso de diferentes tipos de substratos e frequência variada de irrigação no crescimento inicial de pimenta biquinho. Foram utilizadas mudas transplantadas com 45 dias, com o experimento montado em esquema de parcelas subdivididas no delineamento em blocos ao acaso, em esquema fatorial ( $2 \times 2)$, em três repetições e doze parcelas, combinado por dois tipos de irrigação. Irrigação 1 (I1), três vezes ao dia, e Irrigação 2 (I2), duas vezes ao dia, sendo utilizados $100 \mathrm{ml}$ por rega. E conforme o resumo da análise de variância, observou-se que houve efeito significativo $(\mathrm{p}<0,01)$ do substrato (S1) no crescimento inicial de altura e alteração de massa vegetal, comparado ao substrato (S2). No entanto, a frequência variada de irrigação (I1) e (I2) não afetou significativamente os tratamentos. Verificou-se que não houve interação entre os tratamentos com relação aos tipos de substrato (S1) e (S2) x frequência variada de irrigação (I1) e (I2). Comparando-se os tipos de compostos de solo utilizados, concluiu-se que o (S1), composto por substrato, terra vegetal e húmus de minhoca, apresentou melhor desempenho em comparação com o substrato (S2), formado apenas por terra vegetal e húmus de minhoca. Também pode-se observar que a frequência variada de irrigação e sua relação entre os tipos de substratos não afetou significativamente o desenvolvimento vegetal da pimenta biquinho.
\end{abstract}

Palavras-chave: Capsicum chinense; manejo de irrigação; adubação orgânica.

\begin{abstract}
The culture of hot pepper (Capsicum chinense) is of great importance, both for its economic profitability characteristics and its social relevance. Thus, the present study aimed to evaluate the use of different types of substrates and varied frequency of irrigation in the initial growth of pepper pout. Seedlings transplanted at 45 days were used, with the experiment set up in a split-plot design in a randomized block design, in a factorial scheme $(2 \times 2)$, in three replications and twelve plots, combined by two types of irrigation. Irrigation 1 (I1) three times a day and Irrigation 2 (I2) twice a day, using $100 \mathrm{ml}$ per irrigation. According to the summary analysis of variance, it was observed that there was a significant effect $(p<0.01)$ of the substrate (S1) on initial height growth and change in plant mass compared to the substrate (S2). However, the varied frequency of irrigation (I1) and (I2) did not significantly affect the treatments. It was found that there was no interaction between the treatments concerning substrate types $(\mathrm{S} 1)$ and $(\mathrm{S} 2) \mathrm{x}$ varied frequency of irrigation (I1) and (I2). Comparing the types of soil compounds used, it was concluded that (S1) composed of substrate, vegetable earth, and earthworm humus presented better performance compared to the substrate (S2) formed only by vegetable earth and earthworm humus. It was also noted that the varied frequency of irrigation and its relationship between substrate types did not significantly affect the plant development of the pepper pout.
\end{abstract}

Keywords: Capsicum chinense; irrigation management; organic fertilization.

Autor para correspondência: Prof. Dr. Willians Luiz Bueno de Souza, e-mail: willians.souza@unifeb. edu.br, Tel. (17) 98135-0565.

Recebido para publicação: 24/03/2021

Aceito para publicação: $21 / 07 / 2021$

https://doi.org/10.4322/1980-0029.052021 


\section{Introdução}

O cultivo de pimentas no Brasil é de grande relevância, tanto por suas características de rentabilidade, principalmente quando o produtor agrega valor ao produto, quanto por sua importância social. $\mathrm{O}$ alto valor nutricional também as torna um produto diferenciado, por possuírem boas fontes de vitaminas, principalmente $\mathrm{C}$ e $\mathrm{A}$, e por apresentarem vários minerais que são fundamentais para o perfeito funcionamento do organismo. As pimentas possuem propriedades fisiológicas e farmacológicas, além de serem utilizadas como conservante alimentar, devido à presença de determinados componentes, como a capsaicina e a dihidrocapsaicina, em algumas variedades (Zancanaro, 2008; Cisnerospineda et al., 2007).

No mercado brasileiro, as pimentas popularmente conhecidas e apreciadas são as dos tipos malagueta, dedo de moça, de cheiro (ou de bode), americana-doce, cumari (Domenico, 2011). Dentre as demais variedades não tão procuradas pela população, a pimenta de bico começa a ser de interesse de alguns consumidores, devido à inexistência de ardência, que passa a agradar ao paladar de indivíduos das mais diversas faixas etárias, além das propriedades nela existentes que se assemelham às demais (Severo, 2015).

Segundo Ribeiro (2012), o substrato também é um dos fatores que interferem direta e indiretamente para o sucesso do cultivo de pimenteiras em vaso. No mercado há uma diversidade de marcas comerciais que podem ser utilizadas na produção de pimentas envasadas. No entanto, para reduzir os custos de produção, o produtor pode recorrer a um substrato que seja abundante na região e de fácil aquisição, considerando sempre as necessidades de cultivo das pimenteiras. Onofre (2011) descreve que o substrato a ser utilizado deve levar em conta as exigências da semente, como aeração, tamanho, formato, natureza e, principalmente, sua sensibilidade à luz. Com isso o substrato apresenta grande influência na germinação, pois interfere na capacidade de retenção de água e no grau de contaminação por patógenos.

Os estercos são usados frequentemente na composição de substratos, pois têm características propícias à melhoria dos seus atributos físico-químicos, considerados um dos melhores adubos por sua riqueza em nitrogênio, fósforo e potássio (Morais, 2012). Outro componente de importância econômica e ecológica é a casca de arroz carbonizada, em razão da grande disponibilidade da matéria-prima nas regiões produtoras de arroz, como o Maranhão. Algumas pesquisas relacionadas com as propriedades desse material apontam que o mesmo apresenta baixa densidade, $\mathrm{pH}$ próximo da neutralidade, baixa salinidade, elevada porosidade, destacando-se pelo elevado espaço de aeração, baixa retenção de água e manutenção da estrutura no decorrer do cultivo (Souza et al., 2010).

A pimenta é uma das culturas hortícolas mais susceptíveis ao estresse hídrico. Segundo Doorenbos $\&$ Kassam, (2000), precisa-se de 600 a $1250 \mathrm{~mm}$ de água para um ciclo de 120 a 150 dias. De acordo com Marouelli \& Silva (2007), o consumo de água da pimenteira normalmente varia de 500 a $800 \mathrm{~mm}$ por ciclo, ultrapassando até $1000 \mathrm{~mm}$ em cultivares de ciclo longo. Considerando esta sensibilidade ao estresse hídrico, a irrigação é essencial para o bom desenvolvimento da cultura, e o efeito positivo da irrigação tem sido observado em vários trabalhos sobre o efeito do estresse hídrico na redução severa da produção. A deficiência de água, especialmente durante os estádios de floração e formação dos frutos, reduz a produtividade em decorrência da queda de flores e abortamento de frutos (Sezen et al., 2006; González Dugo et al., 2007). Portanto, o presente estudo teve como objetivo avaliar o uso de diferentes tipos de substratos e frequência variada de irrigação no crescimento inicial de pimenta biquinho.

\section{Material e Métodos}

$\mathrm{O}$ experimento foi conduzido em ambiente aberto, no logradouro da Alameda Etiópia, CEP: 14784-057, localizado no município de Barretos-SP, a 425 km da capital do estado de São Paulo, na região Centro-Norte, cujas coordenadas geográficas são: Latitude: $20^{\circ} 33^{\prime} 26^{\prime \prime}$ Sul; Longitude 48 34' 04" Oeste e Altitude média de 530 metros acima do nível do mar (Figura 1).

Na elaboração deste trabalho, foram utilizados 12 vasos com capacidade de 5,6 litros; para os compostos de solo, adotaram-se substrato (turfa, vermiculita, resíduo orgânico agroindustrial e calcário), terra vegetal e húmus de minhoca. Foram determinados dois tipos de preparos de solo, substrato 1 (S1): $50 \%$ substrato, $40 \%$ terra vegetal e $10 \%$ húmus de minhoca; e substrato 2 (S2): $80 \%$ terra vegetal e $20 \%$ húmus de minhoca. 
A cultura utilizada foi a pimenta (Capsicum chinense), cultivar BRS Moema, pertencente ao grupo varietal popularmente conhecido como "biquinho" no estádio de muda transplantada com 45 dias.

O experimento foi montado em delineamento inteiramente ao acaso (DIC), em esquema fatorial (2x2), sendo dois substratos (S1 e S2) e dois tipos de Irrigação (I1 e I2), Irrigação 1 (I1) três vezes ao dia, e Irrigação 2 (I2) duas vezes ao dia, sendo utilizados $100 \mathrm{ml}$ por rega e formado por quatro tratamentos com três repetições, totalizando doze parcelas.

Os vasos com as mudas foram colocados em aparato suspenso do solo em ambiente aberto com espaçamento entre os vasos de $10,0 \mathrm{~cm}$ entre linhas e 20,0 cm entre plantas.

Entre a mensuração dos dados de partida e término do trabalho, decorreram-se 30 dias, realizado no período de 21 de setembro e 20 de outubro de 2019.

Os resultados das características dos tipos de compostos de solo e frequência variada de irrigação foram analisados por estatística descritiva, análise de variância fatorial $(2 \times 2)$ e teste de comparação de médias (Tukey), e para o crescimento inicial altura (cm) e massa (g) da pimenta biquinho avaliada neste estudo utilizando o software BioStat 5.3.

\section{Resultado e Discussão}

Neste estudo foi analisado o uso de dois tipos de substrato (S1) e (S2), frequência variada de irrigação (I1) e (I2) e suas interações, preconizando o crescimento vegetativo inicial através dos valores obtidos da variação (Tabela 1) da altura $(\mathrm{cm})$ e da massa (g) da pimenta biquinho.
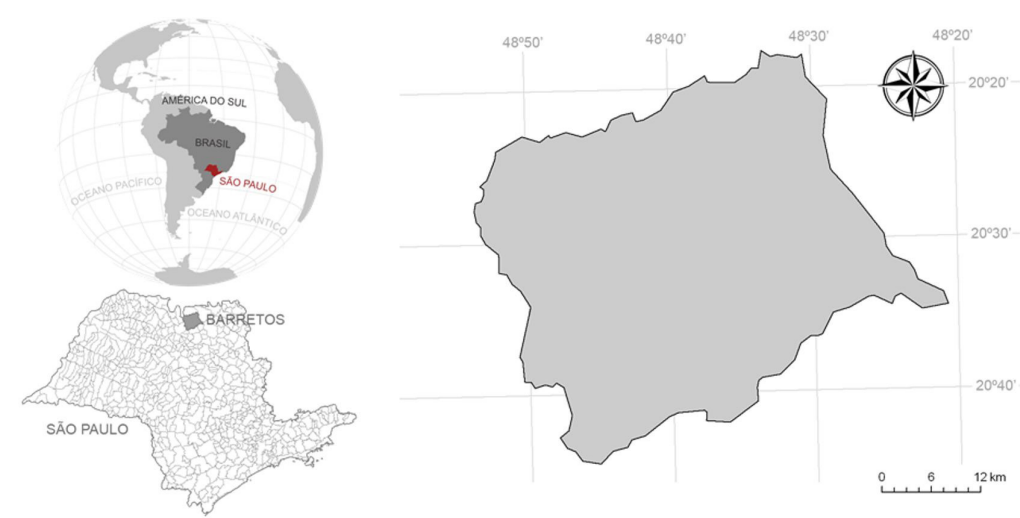

Figura 1. Localização da área de estudo, Barretos, São Paulo, Brasil (Instituto Nacional de Pesquisas Espaciais, 2021).

Tabela 1. Estatística descritiva para o crescimento inicial altura $(\mathrm{cm})$ e massa $(\mathrm{g})$ da pimenta biquinho avaliada neste estudo.

\begin{tabular}{|c|c|c|c|c|c|c|c|c|c|}
\hline Variáveis & $\mathbf{N}^{1}$ & $\begin{array}{l}\text { Média } \\
\text { (cm) }\end{array}$ & $\mathbf{D P}^{2}$ & $\operatorname{Var}\left(\sigma^{2}\right)^{3}$ & Mediana & $\mathbf{E P}^{4}$ & $\begin{array}{l}\mathrm{CV}^{5} \\
(\%)\end{array}$ & Mínimo & Máximo \\
\hline \multicolumn{10}{|c|}{ Altura } \\
\hline S1I1 & 3 & 11,13 & 0,86 & 0,743 & 11,30 & 0,50 & 7,74 & 10,20 & 11,90 \\
\hline S1I2 & 3 & 11,23 & 0,25 & 0,063 & 11,20 & 0,14 & 2,24 & 11,00 & 11,50 \\
\hline S2I1 & 3 & 7,66 & 0,06 & 0,003 & 7,70 & 0,03 & 0,75 & 7,60 & 7,70 \\
\hline S2I2 & 3 & 7,66 & 0,06 & 0,003 & 7,70 & 0,03 & 0,75 & 7,60 & 7,70 \\
\hline \multicolumn{10}{|c|}{ Massa } \\
\hline S1I1 & 3 & 25,63 & 0,58 & 0,34 & 25,40 & 0,34 & 2,30 & 25,20 & 26,30 \\
\hline S1I2 & 3 & 26,27 & 0,37 & 0,14 & 26,10 & 0,22 & 1,44 & 26,00 & 26,70 \\
\hline S2I1 & 3 & 22,20 & 0,17 & 0,03 & 22,30 & 0,10 & 0,78 & 22,00 & 22,30 \\
\hline S2I2 & 3 & 22,23 & 0,35 & 0,12 & 22,20 & 0,20 & 1,58 & 21,90 & 22,60 \\
\hline
\end{tabular}

${ }^{1}$ Número da amostra; ${ }^{2}$ Desvio-padrão; ${ }^{3}$ Variância; ${ }^{4}$ Erro-padrão; ${ }^{5}$ Coeficiente de variação. 
Conforme o resumo da análise de variância para análise fatorial (Tabela 2), observou-se que houve efeito significativo $(\mathrm{p}<0,01)$ do substrato (S1) no crescimento inicial de altura e alteração de massa vegetal, comparado ao substrato (S2). No entanto, a frequência variada de irrigação (I1) e (I2) não afetou significativamente os tratamentos. Verificou-se que não houve inteiração entre os tratamentos com relação aos tipos de substrato (S1) e (S2) x frequência variada de irrigação (I1) e (I2).

$\mathrm{O}$ efeito não significativo da frequência de irrigação sobre a produção da pimenta biquinho também foi apontado por outros autores tais como Aragão (2005) e Gomes (2012), que trabalhando com frequências variadas também não obtiveram resposta significativa para essas variáveis. Lima et al. (2002), avaliando o efeito de irrigação (70,
100 e $130 \%$ da ECA) sobre a cultura do pimentão, constataram efeito significativo para a massa de frutos, com as lâminas apresentando efeito linear crescente, não encontrando o ponto de máximo.

Comparando os tipos de tratamentos substratos x Irrigação por meio do teste de comparação de médias, Tukey a $5 \%$ de probabilidade, observouse a diferença apontada na Tabela 2, apenas para substrato, pois os tratamentos apenas diferiram entre si quando houve a mudança deste fator, sendo apontado como melhor substrato o (S1) $50 \%$ substrato, $40 \%$ terra vegetal e $10 \%$ húmus de minhoca, em relação ao substrato 2 (S2) $80 \%$ terra vegetal e $20 \%$ húmus de minhoca, tanto para crescimento inicial altura $(\mathrm{cm})$ e massa da pimenta biquinho (g) (Tabela 3 ).

Tabela 2. Análise de variância fatorial $(2 \times 2)$ para o crescimento inicial altura $(\mathrm{cm})$ e massa $(\mathrm{g})$ da pimenta biquinho avaliada neste estudo.

\begin{tabular}{|c|c|c|c|c|}
\hline FONTES DE VARIAÇÃO & GL & SQ & QM & $\mathbf{F}$ \\
\hline \multicolumn{5}{|c|}{ Altura (cm) } \\
\hline Substrato & 1 & 37,1008 & 37,1008 & $182,46^{* *}$ \\
\hline Irrigação & 1 & 0,0075 & 0,0075 & $0,037^{\mathrm{ns}}$ \\
\hline Substrato x Irrigação & 1 & 0,0075 & 0,0075 & $0,037^{\mathrm{ns}}$ \\
\hline Resíduo & 8 & 1,62 & 0,20 & --- \\
\hline \multicolumn{5}{|c|}{ Massa $(g)$} \\
\hline Substrato & 1 & 41,8133 & 41,8133 & $261,33 * *$ \\
\hline Irrigação & 1 & 0,3333 & 0,3333 & $2,08^{\mathrm{ns}}$ \\
\hline Substrato x Irrigação & 1 & 0,27 & 0,27 & $1,68^{\mathrm{ns}}$ \\
\hline Resíduo & 8 & 1,28 & 0,16 & --- \\
\hline
\end{tabular}

Tabela 3. Teste de Tukey para o crescimento inicial altura $(\mathrm{cm})$ e massa $(\mathrm{g})$ da pimenta biquinho avaliada neste estudo.

\begin{tabular}{ccc}
\hline TRATAMENTOS & COMPARAÇÕES & DIFERENÇA DE MÉDIAS \\
\hline & Altura & $11,13 \mathrm{a}$ \\
$\mathrm{T} 1$ & $\mathrm{~S} 1 \mathrm{I} 1$ & $11,23 \mathrm{a}$ \\
$\mathrm{T} 2$ & $\mathrm{~S} 1 \mathrm{I} 2$ & $7,66 \mathrm{~b}$ \\
$\mathrm{~T} 3$ & $\mathrm{~S} 2 \mathrm{I} 1$ & $7,66 \mathrm{~b}$ \\
$\mathrm{~T} 4$ & $\mathrm{~S} 2 \mathrm{I} 2$ & \\
& Massa & $25,63 \mathrm{a}$ \\
$\mathrm{T} 1$ & $\mathrm{~S} 1 \mathrm{I} 1$ & $26,27 \mathrm{a}$ \\
$\mathrm{T} 2$ & $\mathrm{~S} 1 \mathrm{I} 2$ & $22,20 \mathrm{~b}$ \\
$\mathrm{~T} 3$ & $\mathrm{~S} 2 \mathrm{I} 1$ & $22,23 \mathrm{~b}$ \\
$\mathrm{~T} 4$ & $\mathrm{~S} 2 \mathrm{I} 2$ &
\end{tabular}

Médias seguidas de mesma letra na coluna não diferem entre si pelo teste de Tukey. 


\section{Conclusão}

A terra vegetal e húmus de minhoca apresentaram melhor desempenho em comparação com o substrato (S2) formado apenas por terra vegetal e húmus de minhoca. A frequência variada de irrigação e a interação com os tipos de substratos não afetaram significativamente o desenvolvimento vegetal da pimenta biquinho.

\section{Referências}

Aragão, V. F. (2005). Produção de pimentão (Capsicum annuum) em diferentes níveis de nitrogênio e lâminas de irrigação (Dissertação de mestrado). Universidade Federal de Campina Grande, Campina Grande.

Cisnerospineda, O., Torres-Tapia, L. W., GutiérrezPacheco, L. C., Contreras-Martín, F., GonzálesEstrada, T., \& Perada-Sánchez, S. R. (2007). Capsaicinoids quantification in chili peppers cultivated in the state of, Yucatan, Mexico. Food Chemistry, (104), 1755-1760.

Domenico, C. I. (2011). Caracterização agronômica e pungência em Pimenta (Capsicum chinense Jacq) (Dissertação de mestrado). Instituto Agronômico, Campinas.

Doorenbos, J., \& Kassam, A. H. (2000). Efeito da água no rendimento das culturas (Estudos FAO: Irrigação e Drenagem, 33, 221 p.). Campina Grande: UFPB.

Gomes, D. P. (2012). Desempenho do consórcio de alface e cenoura, sob manejo orgânico com irrigação automatizada (Dissertação de mestrado). Universidade Federal Rural do Rio de Janeiro, Seropédica.

González-Dugo, V., Orgaz, F., \& Fereres, E. (2007). Responses of pepper to deficit irrigation for paprika a production. Scientia Horticulturae, 114, 77-82.

Instituto Nacional de Pesquisas Espaciais - INPE. Divisão de Processamento de Imagens. (2021). DPI Canasat. Dados do Município de Barretos - SP 2006 a 2011. Recuperado em 24 de março de 2021, de http://www.dsr.inpe.br/laf/canasat
Lima, L. M. L., Fernandes, D. L., Souza, M. W. R., Melo, P. C., Mendonça, F. C., Teodoro, R. E. F., Luz, J. M. Q., \& Carvalho, H. P. (2002). Utilização de diferentes lâminas de irrigação e substratos orgânicos na produção de pimentão. In Anais do $42^{\circ}$ Congresso Brasileiro de Olericultura. Brasília: Sociedade de Olericultura do Brasil. 1 CD-ROM.

Marouelli, W. A., \& Silva, H. R. (2007). Irrigação da pimenteira (Circular Técnica, 51, 14 p.). Brasília: Embrapa/CNPH.

Morais, F. A., Góes, G. B., da Costa, M. E., e Melo, I. G. C., Veras, A. R. R., \& Cunha, G. O. M. (2012). Fontes e proporções de esterco na composição de substratos para produção de mudas de jaqueira. Revista Brasileira de Ciências Agrárias, 7(supl.), 784-789.

Onofre, I. T. M. (2011). Efeito de diferentes subtratos na germinação e vigor de semente canafistula Schizolobium amazonicum Herb. (Caesalpinaceae) (Trabalho de Conclusão de Curso). Universidade Federal do Acre, Rio Branco.

Ribeiro, W. S. (2012). Avaliação de substratos e poda na produção de pimenteira ornamental (Dissertação de mestrado). Universidade Federal de Viçosa, Viçosa.

Severo, D. S. (2015). Farelo de pimenta biquinho (Capsicum chinense) aplicado na produção de Boursin (Trabalho de Conclusão de Curso). Universidade Federal de Campina Grande, Campina Grande.

Sezen, S. M., Yazar, A., \& Eker, O. S. (2006). Efeitos de regimes de irrigação no rendimento e qualidade em campo de pimenta. Agricultural Water Management, 18(1), 115-131.

Souza, A. R. C., Ferraz, R. C., Peiter, M. X., Robaina, A. D., Schwab, N. T., Neuhaus, M., \& Girardi, L. B. (2010). Atributos físicos de misturas entre casca de arroz carbonizada e solo da unidade São Pedro. In Anais do VII Encontro Nacional de Substratos para Plantas. Goiânia: UFG.

Zancanaro, R. D. (2008). Pimentas: tipos, utilização na culinária e funções no organismo (Monografia). Universidade de Brasília, Brasília. 